\title{
Study on the Ultrasonic Application in Chemistry and Chemical Engineering
}

\author{
Yunshen Zhang \\ Department of Petrochemical Engineering, Puyang Vocational and Technical College, puyang \\ Henan, P. R. China 457000
}

\begin{abstract}
Keywords: Ultrasound; Sonochemistry; Ultrasonic cavitation; Waste water treatment; Nanomaterials; Process strengthening
\end{abstract}

\begin{abstract}
With the continuous development and progress of science and technology in China, chemical and chemical industry has made remarkable achievements in recent years, the ultrasonic technology used in chemical and chemical work, research progress has also been a breakthrough achievement. In this paper, combined with the author's practical work experience, focuses on the ultrasonic technology in polymer degradation and polymerization, toxic organic pollutants in water treatment, organic synthesis and ultra-strong transfer process (including adsorption process, extraction process, emulsification and demulsification, crystallization Process, membrane process, heterogeneous chemical reaction process and electrochemical process). In addition, the application of ultrasound in the preparation of nanomaterials and wastewater treatment as well as the application of ultrasonic scale inhibition technology are introduced. The application of ultrasonic technology to the coupling of traditional chemical and chemical preparation process will bring new vitality and technological progress to the chemical industry.
\end{abstract}

Since the reform and opening up, ultrasonic technology has been widely used in many chemical fields, ultrasonic has been widely used in chemistry and chemical industry, such as chemical process intensification, waste water treatment, chemical reaction strengthening and new material synthesis, etc. Accelerated mass transfer and accelerated agitation are the basic principles of the application of ultrasound in chemical and chemical engineering. This principle explains the basic principles of the physical, chemical and hole-induced effects of cavitation. The process of concentrating the sound field energy and releasing the energy is the fundamental reaction of the acoustic cavitation process of the liquid. Liquid in the ultrasonic radiation, it will produce tiny gas bubble, the gas bubble in the surrounding fixed small space, and it can release the high temperature and huge gas pressure in the role of these tiny gas bubble, a fixed space, the temperature with the strong impact of the jet will have a huge jet of energy, these energy accumulation, will release a huge energy in the impact of these energy, can make strong bond with the bond breaking, inevitably produce a series of chemical reactions, Including free radical initiated polymerization, degradation, reduction, substitution, oxidation, molecular fragmentation. Due to the application of ultrasonic wave in chemical and chemical industry, chemical reaction which can not be carried out or difficult to be carried out. The chemical reaction product can be selected and the rate of chemical reaction can be increased, . The application of ultrasound in chemical and chemical industry has a positive and far-reaching influence on the chemical industry, greatly improving the preparation process of chemical products, and in this reaction process, the application of ultrasonic to chemical preparation becomes a more efficient, safe, convenient and simple technology, so that technical strength, shorten the time, ultrasonic technology in chemical and chemical preparation of the application, significantly different from the traditional external heating, stirring and other chemical technology methods. This paper will elaborate on the application of ultrasonic in chemical and chemical industry.

\section{The working principle of ultrasound}

By a series of density of the longitudinal and transverse waves constitute the ultrasonic wave, through the liquid medium can spread to the surrounding. Under the action of the specified power 
ultrasound, the liquid in the ultrasonic wave can be vaporized into gas, which produces a vacuole, which is a small dispersed gas bubble. Under the action of high temperature and high pressure, the tiny gas bubble rapidly explodes. After the explosion, in which a huge local high-pressure and high-temperature environment, in this environment, there may be a high-speed movement of micro-jet, micro-jet under the influence of high-speed effect, the chemical reaction will have a huge mechanical stirring effect, through this effect, the micro-jet will penetrate the laminar boundary layer to limit the chemical reaction between the chemical reaction speed and propagation process. In the chemical process, the application of ultrasonic technology, mainly the application of ultrasonic technology, ultrasonic vaporization in the process of liquefaction into the release of enormous mechanical forces and high chemical reaction force, which generated a huge mechanical force of the main ultrasonic.The surface area of the heterogeneous reaction interface is increased, the heat transfer process is enhanced and the mass transfer occurs in the chemical reaction interface and the eddy current effect, and the high efficiency chemical reaction force is considered as the micro gas bubble in the general sense. Cracking of chemical bonds in cavitation bubbles, pyrolysis, generation of free radicals and radical - related reactions. In general, processes such as emulsification and demulsification, adsorption, crystallization, extraction, sonication scale inhibition, electrochemistry, filtration, suspension separation, membrane processes, heterogeneous chemical reactions, heat transfer and ultrasonic cleaning are considered ultrasonic applications chemical and chemical fields in the common mechanical effects. The chemical effect of the use of the process, including polymer chemistry, organic degradation and other free radical reactions. In practice, the staff often find that the chemical reaction is often a huge mechanical force and efficient chemical reaction force of the two effects of the comprehensive results.

In chemical and chemical applications, ultrasonic efficiency will be affected by many factors, including the form of the reactor, dissolved gas, surface tension, viscosity of the solution temperature, ultrasonic intensity and frequency and $\mathrm{PH}$ value.

\section{The application of ultrasonic technology in chemical industry}

2.1 Substitution reaction. In the field of chemical and chemical engineering, the phenomenon of supersonic technology applied to substitution reaction is very common, most of which have certain commercial value and use value. Ultrasonic technology will be used in the preparation of industrial chemical engineering equipment, by accelerating the reaction can be difficult to break down the material such as wax, fat, oil and other organic matter decomposition, access to low-cost chemical products.

\subsubsection{Hydrolysis reaction}

The experimental study concluded that ultrasonic technology can be applied to the general hydrolysis reaction process, which can effectively moderate chemical reaction conditions, usually, the rate of chemical reaction and the temperature is positively related to the process. For example, nitrobenzene ester hydrolysis reaction in the alkaline environment, with temperature changes, the hydrolysis reaction rate increased significantly, and that the reaction changes with the chemical structure of the molecular structure itself.

In the ultrasonic technology, the nitrile, a chemical derivative, in the alkaline environment, carboxylic acid hydrolysis reaction, the amount of access to a substantial increase in the chemical reaction, without the use of phase transfer catalyst, Except that the reaction mixture was refluxed.

\subsubsection{Alkylation reaction}

In the $\mathrm{N}$-alkylation process, the application of ultrasonic technology, greatly accelerating the reaction time of the crown compound, mild reaction conditions, but also to a certain extent, improve the efficiency of reaction production.

2.2 Solid-liquid extraction strengthening. As a kind of ultrasonic technology, ultrasonic chemical extraction technology has been widely used in analytical chemistry in recent years. It has become a mainstream ultrasonic technique in plastic or food processing and solid sample extraction and preparation of trace components. This solid-liquid extraction strengthening application example is more common in the chemical preparation process, for example, the benzopyrene is extracted 
from the environmental sample to obtain the same amount of product extract as the premise, after the ultrasonic technology is used, the reaction process only 3 minutes, while the traditional preparation process, the Soxhlet extraction method takes about a day time. Ultrasonic chemical extraction technology is applied to agricultural production process, such as the use of plant roots as raw materials, sugar cane sugar can be extracted, effectively shorten the reaction time and improve the reaction yield. Ultrasonic chemical extraction technology used in agriculture, is the use of a small gas bubble in the ultrasound generated micro-jet, resulting in destruction of plant cell wall, the original in the peripheral environment of the plant cytoplasm flow into the surrounding liquid, resulting in plant cell traits, plant nuclei in the ultrasound.Resulting in high strength under the action of cavitation nuclear collapse, leading to complete destruction of plant cells through the destruction of the cell wall reaction to improve the mass transfer process and improve the extraction rate and efficiency. In the pharmaceutical industry, the same can be used ultrasonic technology, advanced Chinese medicine extraction technology, can not be separated from the support of ultrasound technology .

2.3 Desorption strengthening and adsorption. The difference of the solute concentration in the porous material between the solution body and the adsorption surface is the main resistance to form the solute in the diffusion medium, the desorption process during mass transfer and the physical adsorption. Through the desorption and adsorption, the perturbation of the medium in the hole is obviously enhanced, and the mass transfer boundary layer thickness decreases with the increase of the total mass transfer efficiency and rate.

2. 4 Crystallization process. When supersaturated solution forms new phase, under the action of ultrasonic wave, the crystal will receive serious interference, can not increase the quality, along with the nucleation rate is accelerated obviously, the degree of crystal dispersion will be promoted greatly. By changing the physical and chemical properties of crystal particles, the use of ultrasonic technology in the crystallization process has become an essential means to control the properties and state of particles, and is widely used in practical work. When the supersaturation state is not reached, the nucleation process is realized under the influence of the acoustic field force, and the ultrasonic technology has the advantages unmatched by other crystallization techniques in the crystallization process. When the ultrasonic technology is applied, To ensure that the grain distribution more uniform, more smooth, more complete.

2. 5 Emulsification and demulsification. In the coatings industry, food processing industry, heterogeneous liquid-liquid reaction and polymer emulsion polymerization and other chemical industry, the application of ultrasonic technology is equally broad. Emulsification and demulsification are typical examples of applications of ultrasound technology in these fields. To overcome the micro-jet interface in the liquid produced by the interface between the energy in this energy, the strong mixing between the droplets to form highly dispersed emulsion, that is, emulsification and demulsification.

2. 6 Ultrasound Electrochemistry. Ultrasonic electrochemistry is a new discipline, the subject is the combination of electrochemical and ultrasonic technology, the application of these two technologies in the electrochemical synthesis and decomposition and electroplating chemical reaction. In this technique, ultrasound can accelerate the electrochemical reaction by accelerating the current efficiency in the chemical reaction, reducing the over-potential and so on. Ultrasonic electrochemistry in the production of chemical and chemical products in the production process, greatly enhanced the rate of chemical reaction, ultrasonic applications in the electrochemical reaction process, resulting in a positive positive effect. The results of microscopic mixing and cavitation of the electrode surface are fundamental principles of the combination of electrochemical and ultrasonic techniques for their application.

Through the ultrasonic dielectric effect, ultrasonic solid or liquid substances contained in some of the electromagnetic energy into heat, resulting in chemical reactions can cause the heat, known as the principle of ultrasonic heating. The core of this principle is the role of the ultrasonic, polar chemical reaction, so that chemical molecules with the electric field direction is consistent. In the electric field, the positive and negative electric field changes, the electrode in the transition process, 
the polarity of the compound with the positive and negative changes in the electrode to meet the orientation and consistency of the external field. Under the driving of the ultrasonic heating electric energy, the chemical molecules are in the state of changing the polarity of the electric field, and turn back and forth, the torsion effect will occur, the internal temperature of the compound will rise rapidly, and the effect of enhancing the chemical reaction speed will be exerted.

2.7 The application of ultrasonic technology in organic synthesis. In the 1960s, in the organic chemistry synthesis reaction, appeared the use of electromagnetic radiation pulse organic synthesis example, ultrasonic radiation in acrylic acid, acrylic acid ester and methacrylic acid in the emulsion polymerization has been applied. In 1986, foreign scientists will be truly applied to organic synthesis of ultrasound technology, the use of ultrasonic radiation to accelerate chemical reactions, can significantly enhance the rate of chemical reaction, the reaction products with the substantial increase. Moreover, the ultrasonic technology used in organic synthesis, but also improve product quality and recovery, save energy and improve efficiency.

2. 8 Ultrasonic degradation of wastewater. For the organic pollutants that are difficult to be degraded, it can also be used to hydrolyze chemical pollutants by ultrasonic wave. The technology to incineration, supercritical oxidation and advanced oxidation and other advantages of a variety of water treatment technology, with a wide range of applications, the degradation rate is fast, the conditions of mild degradation and so on. Is a promising application and development potential of new technologies, ultrasonic treatment of sewage degradation technology can be used alone or in combination with other water treatment technology.

2. 9 Scale inhibition. When the ultrasonic wave is applied to the boiler scale, it can effectively avoid boiler scaling. First, the micro-jet generated by ultrasonic wave can rapidly scour the surface of the boiler under the strong stimulating force to ensure that the precipitated matter produced during the reaction process is not easy to attach, and the contact area is large, Ultrasonic energy can be deposited and penetrated materials, in the ultrasonic action, the heat can be spread evenly to the various parts of the material to achieve rapid and uniform to ease the solid surface into the scale of the pressure, to eliminate the supersaturation of the solution.

Application of ultrasound in chemical and chemical engineering is a newly established interdisciplinary, which plays an active role in the chemical and mechanical effects produced by ultrasonic cavitation. When the ultrasonic wave is correctly applied to chemical industry, it is necessary to correctly understand the interaction between matter and ultrasonic according to the characteristics of chemical industry, so that we can study more deeply. So ultrasonic technology can be applied to any process and system of process rate controlled by interface effect, and cooperate with traditional technology to better promote the development of new market of chemical technology in our country. Therefore, the application of ultrasonic technology in chemical industry is developing rapidly.

\section{References}

[1] Kong Shenglu, Gong Changsheng.Studies on sound and chemical engineering [J]. Modern Chemical Industry, 2009, 9 (6): 23-27.

[2] Zhao Yiyun, Bao Cikuang, Feng Ruo.New advances in applied research of sonochemistry [J]. Chinese Journal of Chemistry, 20144, 8: 26-29.

[3] Yao Weixi, HE Yu-Lian, LI Yuqin.Extraction of Benzo (a) pyrene in Environmental Samples by Ultrasonic Irradiation [J]. Environmental Science, 2012, 3 (1): 18-21.

[4] Guo Xiaowu, Zhang Fucheng, Lin Shuyu, etc.Experimental study of ultrasonic extraction of berberine [J]. Journal of Shaanxi Normal University, 2011, 22 (1): 89- 92.

[5] Qin Wei, Yuan Yonghui, Dai Youyuan .Enhancement of Chemical Separation Process in Ultrasonic Field [J] Chemical Industry and Engineering Progress, 2014, 1: 1-5. 